\title{
Thick (3D) Sample STEM Imaging at Nano Scale: iDPC and ADF Simultaneously
}

Eric G.T. Bosch ${ }^{1}$, Ivan Lazić ${ }^{1}$, Emrah Yücelen ${ }^{1}$, Piet Trompenaars ${ }^{1}$

1. Thermo Fisher Scientific, Materials \& Structural Analysis, Eindhoven, The Netherlands.

Depth sectioning STEM imaging is one of the approaches to reveal structural 3D sample information at atomic and nano scale. Weather it is used as confocal [1] or standard (HA)ADF [2, 3] or (A)BF [4] STEM 3D information is obtained by placing the crossover of the probe at various depths of the sample by setting different probe defocus values, thereby obtaining a through focus series (TFS) scan in depth through the thick sample of interest.

Although relatively easy to perform experimentally, TFS of conventional STEM produces images for which interpretation has been a challenge, due to nonlinearity in the contrast transfer, like in (A)BF [5, 6] or channelling, affecting ADF STEM.

Here we introduce depth sectioning using iDPC-STEM $[7,8]$ and reveal three dimensional structural information of various samples of different thicknesses containing nano scale size features. We will show that, although the outcome is relatively easy to interpret, at this scale additional effects occur that influence the imaging process. In order to reveal and explain the origin of these effects we perform simultaneous TFS iDPC-STEM with TFS ADF-STEM. Resolution in z direction, as well as theoretical interpretation based on simulations and theory [6] will be discussed.

In Fig. 1 an example of TFS iDPC-STEM imaging is presented. A sample consisting of a $20 \mathrm{~nm}$ thick crystal of Si [110] at which, from both sides, $\sim 10 \mathrm{~nm}$ diameter Pt nanoparticles were deposited was imaged. Although the side view of the TFS iDPC-STEM image stack nicely shows particles on both sides of the Si crystal (right part of Fig. 1), the top view image at a defocus corresponding to the optimal ADF-STEM image shows defocused but also contrast inverted particles at the bottom of the sample.

Figure 2 displays another example used to further study this contrast reversal effect. Gold nano particles on a carbon substrate are imaged using simultaneous TFS iDPC/ADF-STEM. Three depth positions are shown in the left part of the image, focusing the sample (left) at the center of the sample (middle) and at the bottom part of the sample (right). The side views (Fig. 2 right) shows both the $\sim 5 \mathrm{~nm}$ diameter crystalline gold particles and the amorphous carbon layer of about $20 \mathrm{~nm}$ thick.

\section{References:}

[1] J.J. Einspahr, P.M. Voyles, Ultramicroscopy 106 (2006), p. 1041-1052.

[2] G. Behan et al, Phil. Trans. R. Soc. A 367 (2009), p. 3825-3844.

[3] G. Saito et al, Ultramicroscopy 175 (2017), p. 97-104.

[4] S.D. Findlay et al, Ultramicroscopy 110 (2010), p. 903-923.

[5] E.G.T. Bosch \& I. Lazić, Ultramicroscopy 156 (2015), p. 59-72.

[6] I. Lazić, E.G.T. Bosch, Advances in Imaging and Electron Physics 199 (2017), p. 75-184.

[7] I. Lazić, E.G.T. Bosch and S. Lazar, Ultramicroscopy 160 (2016), p. 265-280.

[8] E. Yücelen, I. Lazić, E.G.T. Bosch, Scientific Reports 8 (2018), p. 2676. 


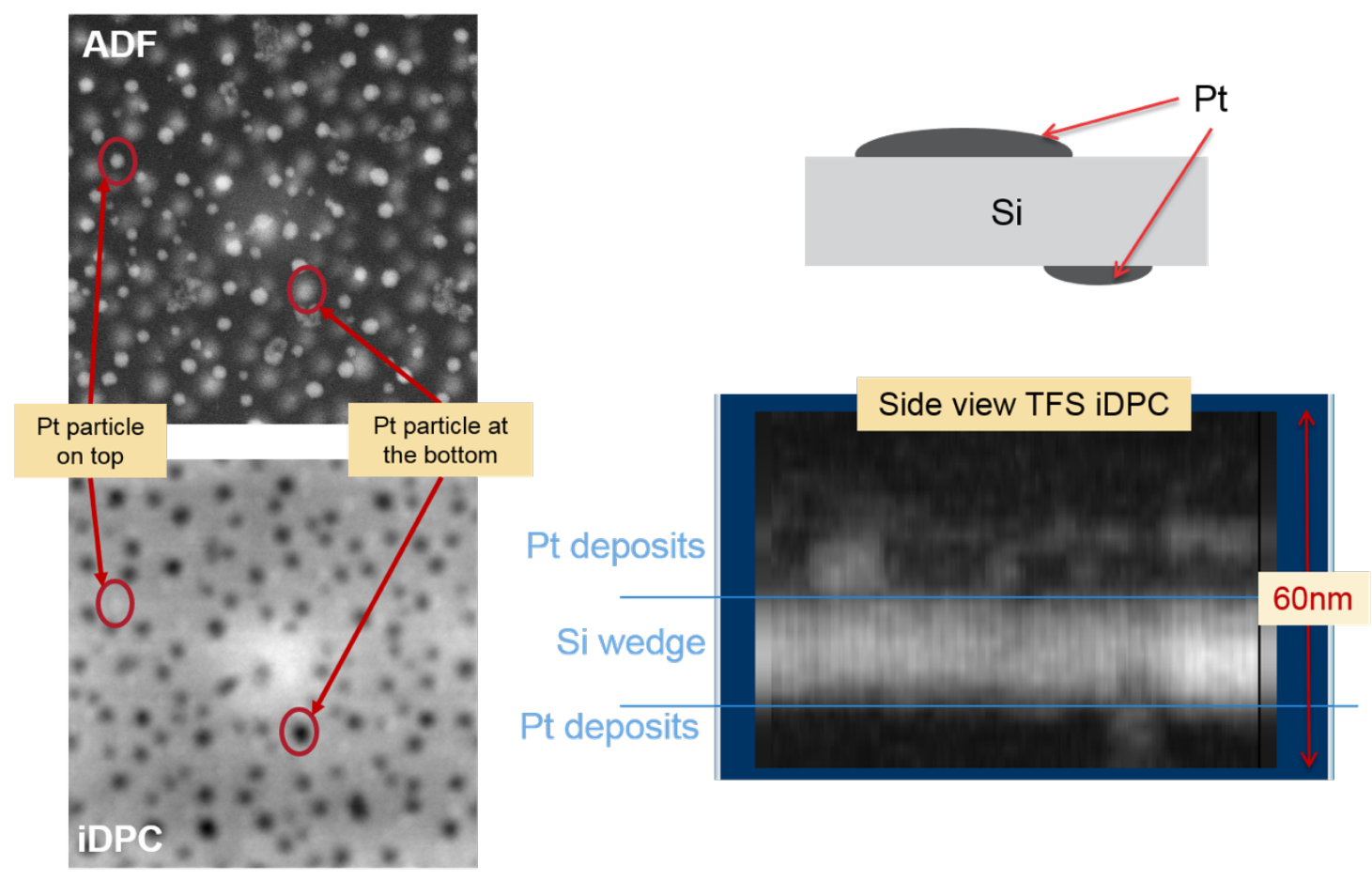

Figure 1. Si [110] crystal of $20 \mathrm{~nm}$ thickness with $\sim 10 \mathrm{~nm}$ diameter Pt nanoparticles deposited at both sides. Left: Simultaneous ADF-STEM (top) and iDPC-STEM (bottom) image at defocus chosen such that the ADF image is overall the sharpest. Right: side view of the TFS (depth sectioning) iDPC-STEM stack shown along the line connecting the two particles indicated in the left part.

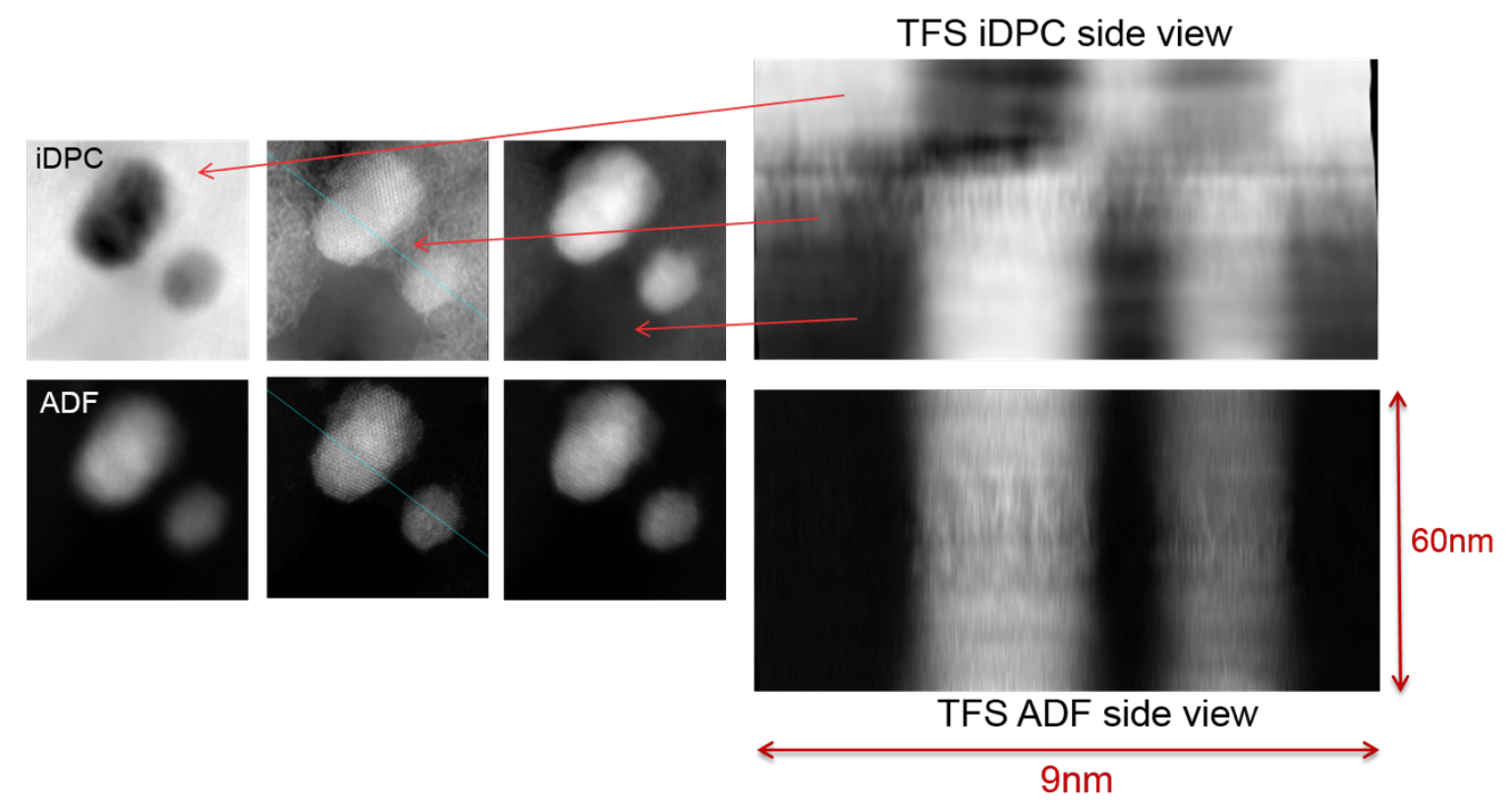

Figure 2. Simultaneous TFS iDPC/ADF-STEM imaging of gold nano particle on a carbon substrate. Left: Three depth positions, above (left) at the middle (middle) and below the sample (right). Right: A side view of TFS iDPC-STEM (top) where contrast reversal is clearly visible and side view of TFS ADF-STEM (bottom) where contrast reversal is absent. 